\title{
3 Research Square

\section{High Incidence, Re-Infections, and Active Syphilis in Populations Attending A Specialized HIV Clinic in Mexico, A Dynamic Cohort Study.}

Omar David Tumalan-Gil

Instituto Nacional de Salud Publica

Veronica Ruiz-González

Clinica Especializada Condesa

Santa García-Cisneros

Instituto Nacional de Salud Publica

Andrea González-Rodríguez

Universidad Nacional Autonoma de Mexico Coordinacion de Humanidades

Antonia Herrera-Ortiz

Instituto Nacional de Salud Publica

Maria Olamendi-Portugal

Instituto Nacional de Salud Publica

Miguel Sanchez-Aleman ( $\boldsymbol{M}$ msanchez@insp.mx )

Instituto Nacional de Salud Publica https://orcid.org/0000-0003-2383-7930

\section{Research Article}

Keywords: syphilis, syphilis serodiagnosis, cohort studies, incidence.

Posted Date: June 3rd, 2021

DOl: https://doi.org/10.21203/rs.3.rs-559014/v1

License: (c) (i) This work is licensed under a Creative Commons Attribution 4.0 International License.

Read Full License 


\section{Abstract}

Background. Syphilis has reemerged in many vulnerable groups around the world. The objective of the current study was to determine the prevalence and incidence of syphilis among people who attended a specialized HIV clinic in Mexico from 2011-2015.

Methods. Databases from the laboratory were analyzed and four groups were formed: people seeking HIV-1 voluntary counseling and testing (VCT), people in prison (PPr), people living with HIV (PLWH) and patients from primary care clinics (others). Syphilis diagnosis was made using the reverse algorithm; antibody titers were examined to determine the stage of infection. Baseline data was analyzed, and with follow-up information, a retrospective dynamic cohort was formed. Factors associated with seroprevalence of syphilis and active syphilis were evaluated by the chi-square test. Moreover, risk factors for the incidence of syphilis were described.

Results. A total of 81,863 baseline individuals were analyzed. Syphilis seroprevalence was $9.9 \%$ in VCT, $8.2 \%$ in $\mathrm{PPr}, 37.0 \%$ in PLWH, and $8.7 \%$ in others; the prevalence of active syphilis was $1.7-13.1 \%$. A total of 11,124 people were followed-up; the incidence (cases per 100 years people) was 3.5 among VCT and 16.0 among PLWH; moreover, the frequency of re-infections was $11.1-24.4 \%$. Men, transgender, persons between 20-39 years old, and individuals with a history of HIV or hepatitis B had a higher risk of syphilis.

Conclusions. Several vulnerable groups have exhibited a reemergence of syphilis, with high prevalence of active syphilis, high incidence, and re-infections. Persons living with HIV had the highest risk.

\section{Background}

The World Health Organization (WHO) estimated the number of new cases of syphilis in 2012 at 5.6 million; it increased to 6 million in 2016. In the latter year, the incidence was 1.7 cases per thousand women and 1.6 cases per thousand men. Globally, the syphilis prevalence was $3.2 \%$ among female sex worker (FSW) and 6.0\% among men who have sex with men (MSM). The WHO set the goal to reduce the incidence of Treponema pallidum by $90 \%$, with a focus on priority groups: MSM, FSW, and intravenous drug users (IDUs). ${ }^{1,2}$

Syphilis has increased in different parts of the world. Between 2013 and 2017, the number of cases per 100,000 inhabitants in the U.S. increased from 5.5 to $9.5 .^{3}$ The European Economic Community reported an increase from 4.7 cases to 6.1 cases per 100,000 inhabitants between 2012 and 2016. ${ }^{4}$ In Mexico, an increase in syphilis was documented among young men, focused in Mexico City. ${ }^{5}$ Syphilis is important because of the number of cases as well as the higher risk to acquire and transmit HIV. Moreover, syphilis and HIV coinfection negatively modifies both diseases. ${ }^{6}$

The Clinica Especializada Condesa (CEC) in Mexico City is the institution that serves the largest number of people seeking HIV testing, as well as people living with HIV. The CEC also performs test for other sexually transmitted infections (STI), hepatitis B virus (HBV), hepatitis C virus (HCV), and syphilis. The 
objective of the current study was to determine the prevalence, incidence, and risk factors of infection by T. pallidum among populations attending the CEC from 2011-2015.

\section{Methods}

A database was elaborated with the variables year, internal number, population type, sex, age, HIV by chemiluminescent microparticle immunoassay (CMIA), HIV rapid test 1, HIV rapid test 2, HIV Western blot, T. pallidum by CMIA, T. Pallidum rapid test, venereal disease research laboratory (VDRL) titer, HVB antigen detection, and HCV antibodies detection. Syphilis was detected using the reverse algorithm. This method starts with a treponemal test to later perform the VDRL. Some authors have mentioned that in the absence of clinical data, the antibody titer can be used to determine the stages of syphilis, as active syphilis (titer $\geq 1: 8$ ), latent syphilis (titer 1:1-1:4) and cured syphilis (VDRL negative, positive treponemal test). ${ }^{7,8}$ The attending population was classified into four groups: (1) people seeking HIV-1 voluntary counseling and testing (VCT), (2) people living with HIV (PLWH), (3) people in prison (PPr), and (4) others, people from others clinics, principally primary care clinics. The CEC sends patients with syphilis to their primary care clinics for treatment, only PLWH without social security attended CEC. The present study was approved by the ethics committee of the National Institute of Public Health from Mexico. The database had identification keys, we did not have access to the names of the participants.

From the baseline information, frequency analysis stratified by population group was performed, and chisquare $\left(\mathrm{X}^{2}\right)$ tests were used to evaluate the differences. Subsequently, a bivariate analysis was performed to determine associated variables, both to the seroprevalence of syphilis and the presence of active syphilis. The trend test was used for the year and age variables, considering the four population groups.

A retrospective dynamic cohort was elaborated with individuals that had two syphilis tests with at least four months between measurements and incidence densities was calculated using person years (py). During follow-up, syphilis was considered cured if the antibody titer decreased until negative or if the antibody concentration decreased four times or more. Syphilis re-infection was defined when it was cured at baseline and the titer was $\geq 1: 1$ during follow-up or if antibody titer increased four times or more. Persistent infection was considered if the antibody titer remained similar ( \pm 2 times). ${ }^{9}$ Incidence rate ratios (IRR) were calculated with $95 \%$ confidence intervals $(\mathrm{Cl})$, and a cumulative incidence curve was constructed using the Kaplan-Meier method. A p-value $<0.01$ was considered statistically significant. Statistical analysis was performed using the SPSS program.

\section{Results}

Basal. A total of 129,613 blood/serum samples were processed in the CEC laboratory between 2011 to 2015. In 2011, there were 17,589 samples, and in 2015, there were 37,342 . Results for syphilis detection were from 95,732 samples, of which 81,863 people attended at least once; $78.1 \%$ were VCT, 9.9\% PPr, $2.2 \% \mathrm{PLWH}$, and $9.8 \%$ others. Table 1 shows the demographic characteristics and laboratory results of the four analyzed populations. In all groups, there was an increase in the number of samples analyzed 
over the years, with the exception of PPr that presented the greatest number of samples during 20122013. Men were the majority in all groups, from $63.3 \%$ among others to $85.5 \%$ among PLWH. The highest percentage of transgender was in the PLWH group. PLWH had the highest average age at 34.6 years, and VCT were the youngest on average (29.0 years). One-in-five VCT individuals were HIV positive; $3.8 \%$ of PPr individuals were HIV positive. Among PLWH, 4.9\% had HBV antigens and $2.9 \%$ had antibodies against HCV. 
Table 1

Demographic and clinical characteristics in populations attending a specialized HIV clinic in Mexico.

\begin{tabular}{|c|c|c|c|c|c|}
\hline & $\begin{array}{l}\text { VCT } \\
N=63,916\end{array}$ & $\begin{array}{l}\text { PDL } \\
N=8,083\end{array}$ & $\begin{array}{l}\text { PLWH } \\
N=1,808\end{array}$ & Others $\mathrm{N}=8,056$ & $\mathbf{p}$ \\
\hline & $\%$ & $\%$ & $\%$ & $\%$ & \\
\hline \multicolumn{6}{|l|}{ Year } \\
\hline 2011 & 8.9 & 16.1 & 18.8 & 13.3 & $<0.001$ \\
\hline 2012 & 19.3 & 25.3 & 24.5 & 17.5 & \\
\hline 2013 & 21.9 & 22.3 & 19.1 & 24.5 & \\
\hline 2014 & 23.4 & 17.3 & 14.8 & 23.3 & \\
\hline 2015 & 26.5 & 19.0 & 22.8 & 21.5 & \\
\hline \multicolumn{6}{|l|}{ Sex } \\
\hline Female & 34.3 & 31.8 & 11.9 & 36.6 & $<0.001$ \\
\hline Male & 64.3 & 67.2 & 85.5 & 63.3 & \\
\hline Transsexual & 1.3 & 1.0 & 2.6 & 0.1 & \\
\hline \multicolumn{6}{|l|}{ Age (years) } \\
\hline$\leq 9$ & 1.1 & 0.1 & - & 7.9 & $<0.001$ \\
\hline $10-19$ & 11.7 & 2.4 & 2.0 & 19.2 & \\
\hline $20-29$ & 49.9 & 33.7 & 32.2 & 28.7 & \\
\hline $30-39$ & 22.0 & 37.9 & 36.8 & 19.8 & \\
\hline $40-49$ & 9.9 & 18.8 & 21.7 & 12.4 & \\
\hline $50-59$ & 3.9 & 5.9 & 6.1 & 6.8 & \\
\hline$\geq 60$ & 1.5 & 1.3 & 1.1 & 5.1 & \\
\hline \multicolumn{6}{|l|}{ HIV test } \\
\hline Negative & 81.4 & 91.0 & - & 86.0 & $<0.001$ \\
\hline Positive & 18.6 & 3.8 & 100 & 7.2 & \\
\hline Not done & - & 5.2 & - & 6.7 & \\
\hline
\end{tabular}

VCT: People seeking HIV-1 voluntary counseling and testing; PPr: people in prison; PLWH: people living with HIV; Others: patients from primary care clinics. Bold: $p<0.01$ statistically significant. 


\begin{tabular}{|llllll|}
\hline & VCT & PDL & PLWH & Others $\mathbf{N}=8,056$ & P \\
& $\mathbf{N = 6 3 , 9 1 6}$ & $\mathbf{N = 8 , 0 8 3}$ & $\mathbf{N = 1 , 8 0 8}$ & & \\
\hline Negative & 89.1 & 74.2 & 77.3 & 83.9 & $<0.001$ \\
\hline Positive & 0.9 & 0.9 & 4.9 & 0.6 & \\
\hline Not done & 10.0 & 24.9 & 17.8 & 15.5 & $<0.001$ \\
\hline HCV test & & & & & \\
\hline Negative & 95.4 & 81.5 & 81.7 & 86.0 & \\
\hline Positive & 0.9 & 3.1 & 2.9 & 1.4 & 12.6 \\
\hline Not done & 3.6 & 15.4 & 15.4 & & \\
\hline $\begin{array}{l}\text { VCT: People seeking HIV-1 voluntary counseling and testing; PPr: people in prison; PLWH: people } \\
\text { living with HIV; Others: patients from primary care clinics. Bold: } p<0.01 \text { statistically significant. }\end{array}$ \\
\hline
\end{tabular}

The seroprevalence of syphilis was 8.2\% (95\% Cl 7.6-8.8) among PPr, 8.7\% (95\% Cl 8.1-9.3) among others, $9.9 \%(95 \% \mathrm{Cl} 9.7-10.1)$ in the VCT population, and $37.0 \%(95 \% \mathrm{Cl} 34.8-39.2)$ among PLWH. Figure 1 shows the seroprevalence of syphilis and active syphilis. Almost $40 \%$ of people with antibodies showed a cured infection, except among PLWH, in which the percentage was $32.4 \%$. The VCT and PLWH populations had the highest seroprevalence of active syphilis, with 3.6 and $13.1 \%$, respectively.

The seroprevalence of syphilis decreased over time in the CVT and PPr groups $(p<0.001)$. The transgender population had the highest seroprevalence; it was higher among the PPr followed by PLWH, with more than $40 \%$ in both cases $(p<0.001)$. At an older age, the seroprevalence of syphilis increased in the four analyzed population groups $(p<0.001)$. Individuals with other STIs (HIV, HBV, or HCV) had a higher association with the seroprevalence of syphilis $(p<0.05$; Table 2, left).

When analyzing the frequency of active syphilis, there was a decrease during the study period in the VCT group. In contrast, active syphilis increased among PLWH during the analyzed period. Women presented the lowest proportion of active syphilis in the four groups; the highest frequency of active syphilis was in transgender and men living with HIV: $17.0 \%$ and $14.2 \%$, respectively $(p<0.001)$. People aged $20-29$ and 30-39 years had the highest frequency of active syphilis in the VCT group. A history of STIs was associated with a higher frequency of active syphilis in the entire population (Table 2, right). 
Table 2

Seroprevalence of T. pallidum and active syphilis in populations attending a specialized HIV clinic in Mexico.

Seroprevalence T. pallidum

VCT
PPr

\section{PLWH}

Others

\section{Active syphilis}

\section{Year}

$\begin{array}{lllllllll}2011 & 13.2 & 9.9 & 37.5 & 2.0 & 4.7 & 1.8 & 4.7 & 2.0 \\ 2012 & 9.7 & 7.7 & 38.8 & 2.5 & 3.6 & 1.4 & 13.5 & 2.5 \\ 2013 & 9.5 & 9.2 & 33.0 & 2.0 & 3.4 & 1.8 & 15.7 & 2.0 \\ 2014 & 9.2 & 8.9 & 38.4 & 1.9 & 3.1 & 1.6 & 14.6 & 1.9 \\ 2015 & 9.9 & 5.9 & 37.0 & 2.4 & 3.8 & 1.9 & 16.2 & 2.4 \\ \text { Trend p } & <\mathbf{0 . 0 0 1} & \mathbf{0 . 0 0 5} & 0.812 & 0.700 & <0.001 & 0.775 & <0.001 & 0.700 \\ \text { Sex } & & & & & & & & \end{array}$

Female

1.7

7.2

14.9

2.3

0.4

0.8

3.7

2.3

Male

14.1

8.2

39.9

2.1

5.3

1.9

14.2

2.1

transsexual

18.9

43.9

42.6

0.0

5.0

13.4

17.0

0.0

$\mathrm{p}$

$<0.001<0.001<0.001$

0.720

$<0.001$

$<0.001$

$<0.001$

0.720

\section{Age (years)}

$\leq 9$

0.7

33.3

0.9

0.0

0.0

0.9

10-19

3.0

5.7

13.9

1.6

1.8

2.6

13.9

1.6

20-29

9.0

7.5

29.0

2.8

4.0

2.1

14.8

2.8

30-39

13.5

8.1

39.9

1.6

4.6

1.5

13.5

1.6

40-49

12.9

8.4

42.0

2.6

3.1

1.1

11.5

2.6

50-59

13.1

10.2

48.6

2.5

2.1

1.5

9.0

2.5

$\geq 60$

18.2

22.3

52.6

2.7

1.4

2.9

0.0

2.7

$p$ trend $<0.001$

$<0.001$

$<0.001$

0.021

$<0.001$

0.217

0.223

0.021

HIV test

Negative

5.1

6.5

0.8

1.8

1.2

$-$

0.8

Seroprevalence of antibodies against T. pallidum. VCT: People seeking HIV-1 voluntary counseling and testing; PPr: people in prision; PLWH: people living with HIV; Others: patients from others clinics. Active syphilis, titters $\geq 1: 8$ in non-treponemic tests. Bold: $p<0.01$ statistically significant 


\begin{tabular}{|c|c|c|c|c|c|c|c|c|}
\hline \multirow[b]{2}{*}{ Positive } & \multicolumn{4}{|c|}{ Seroprevalence T. pallidum } & \multicolumn{4}{|c|}{ Active syphilis } \\
\hline & 30.8 & 29.4 & 37.0 & 7.9 & 11.4 & 10.7 & 13.1 & 7.9 \\
\hline$p$ & $<0.001$ & $<0.001$ & - & $<0.001$ & $<0.001$ & $<0.001$ & - & $<0.001$ \\
\hline \multicolumn{9}{|l|}{ HBV test } \\
\hline Negative & 9.9 & 7.9 & 34.0 & 1.3 & 3.6 & 1.8 & 11.3 & 1.3 \\
\hline Positive & 41.9 & 34.3 & 49.4 & 13.3 & 17.2 & 10.0 & 21.3 & 13.3 \\
\hline $\mathrm{p}$ & $<0.001$ & $<0.001$ & $<0.001$ & $<0.001$ & $<0.001$ & $<0.001$ & $<0.001$ & $<0.001$ \\
\hline \multicolumn{9}{|l|}{ HCV test } \\
\hline Negative & 9.9 & 7.8 & 32.8 & 1.4 & 3.6 & 1.9 & 11.5 & 1.4 \\
\hline Positive & 26.6 & 12.6 & 53.8 & 3.6 & 9.5 & 1.6 & 19.2 & 3.6 \\
\hline $\mathrm{p}$ & $<0.001$ & 0.003 & $<0.001$ & $<0.001$ & $<0.001$ & 0.030 & $<0.001$ & $<0.001$ \\
\hline
\end{tabular}

Dynamic Retrospective Cohort. There were samples from 11,124 individuals with a baseline syphilis outcome and at least one additional visit to the CEC, the median time between visits was 10.6 months (interval interquartil 6.0-18.0). Figure 2 shows that from baseline, 10,844 people were negative for the treponemal test and 283 were positive. During follow-up of negative samples, 413 positive samples were detected in the second measurement. Considering the non-treponemal test at baseline and second measurement, $59.6 \%$ had a cured infection, $13.6 \%$ had re-infection, and $26.8 \%$ had persistent infection among the VCT group; $29.6 \%$ had a cured infection, $11.1 \%$ re-infection, and $59.3 \%$ persistent infection among PPr; $56.1 \%$ had cured infection, $24.4 \%$ re-infection, and $19.5 \%$ persistent infection among PLWH; $50.0 \%$ had cured infection, $21.4 \%$ re-infection and $28.6 \%$ persistent infection in the others group. Considering all groups, $49.4 \%$ of persons with persistent syphilis infection had twelve months or more between the two measurements (treatment failure); among people without HIV this percentage was $41.9 \%$ (26/62) and among people living with HIV it was 73.7\% (14/19), this difference was statistically significant $(p=0.019)$.

The highest incidence of syphilis was found in the PLWH group, with 16.0 cases per 100 py $(95 \% \mathrm{Cl}$ 12.6-20.3), followed by the VCT population, with 3.5 cases (95\% Cl 3.1-3.8), PPr group with 0.95 cases (95\% $\mathrm{Cl} 0.5-1.7)$, and finally others with 0.86 cases (95\% $\mathrm{Cl} 0.3-2.6)$. Among the PLWH, at 39.67 months (95\% $\mathrm{Cl} 30.91-48.41), 50 \%$ of the population acquired syphilis, a value that was lower compared to VCT individuals (52.5 months; $95 \% \mathrm{Cl} 48.51-56.5)$. This difference was statistically significant $(p<0.001 ;$ Log 
Rank; Fig. 3). For the other groups, it was impossible to calculate the infection time due to the sample size and the low proportion of positive cases.

Table 3 shows the incidence rate ratio for syphilis. Notably, the incidence increased in relation to the calendar year, but this change was not statistically significant. Men and transgender had an incidence of 4.6 cases per 100 py and had a 9-times greater risk to have syphilis compared to women. The 20-29 and 30-39 year age groups had the highest incidence of T. pallidum infection, with 3.9 and 3.4 cases per 100 py, respectively. These groups also had twice the risk of infection compared to individuals over 50 years old. People with a history of HIV infection had an incidence of 12.2 cases per 100 py, those with a history of HBV infection had an incidence of 11.4 cases, and individuals with HCV infection showed 1.7 cases per 100 py. 
Table 3

Incidence and risk factors for T. pallidum infection in populations attending a specialized HIV clinic in Mexico.

\begin{tabular}{|c|c|c|c|}
\hline & Cases/years follow-up & Incidence per 100 py* & IRR** \\
\hline \multicolumn{4}{|l|}{ Year } \\
\hline 2015 & $10 / 309$ & 3.23 & $1.03(0.47-2.02)$ \\
\hline 2014 & $70 / 1,867$ & 3.75 & $1.20(0.84-1.70$ \\
\hline 2013 & $126 / 3,288$ & 3.83 & $1.22(0.90-1.67)$ \\
\hline 2012 & $140 / 4,238$ & 3.30 & $1.05(0.78-1.43)$ \\
\hline 2011 & $67 / 2,137$ & 3.14 & 1.0 \\
\hline \multicolumn{4}{|l|}{ Sex } \\
\hline Male & $386 / 8,411$ & 4.59 & $8.68(5.35-15.06)$ \\
\hline Transgender & $10 / 213$ & 4.69 & 8.88 (3.64-20.54) \\
\hline Female & $17 / 3,217$ & 0.53 & 1.0 \\
\hline \multicolumn{4}{|l|}{ Age (years) } \\
\hline$\leq 9$ & $0 / 51$ & - & \\
\hline $10-19$ & $31 / 956$ & 3.24 & $1.82(0.79-4.91)$ \\
\hline $20-29$ & $244 / 6,267$ & 3.89 & $2.19(1.05-5.51)$ \\
\hline $30-39$ & $99 / 2,951$ & 3.35 & $1.89(0.88-4.82)$ \\
\hline $40-49$ & $31 / 1,120$ & 2.77 & $1.56(0.67-4.19)$ \\
\hline$\geq 50$ & $7 / 394$ & 1.78 & 1 \\
\hline \multicolumn{4}{|l|}{ HIV basal test } \\
\hline Positive & $113 / 923$ & 12.24 & $4.42(3.53-5.51)$ \\
\hline Negative & $298 / 10,767$ & 2.77 & 1.0 \\
\hline \multicolumn{4}{|c|}{ HBV basal test } \\
\hline Positive & $9 / 78$ & 11.54 & $3.21(1.46-6.17)$ \\
\hline Negative & $343 / 9,554$ & 3.59 & 1.0 \\
\hline \multicolumn{4}{|c|}{ HCV basal test } \\
\hline Positive & $2 / 114$ & 1.75 & $0.5(0.1-1.82)$ \\
\hline Negative & $386 / 11,009$ & 3.51 & 1.0 \\
\hline
\end{tabular}


*py: persons years of follow-up; bold: statistically significant. **IRR: Incidence rate ratio

\section{Discussion}

The prevalence of syphilis in the groups that attended the CEC is high (8.2\% among PPr to $37.0 \%$ among PLWH) compared to other vulnerable populations such female sex workers $(14.2 \%),{ }^{10}$ people in prison $(2.0 \%),{ }^{11}$ and MSM $\left(9.5 \%, 14.8 \%\right.$, and $30 \%$ in 2014, 2016, and 2017, respectively). ${ }^{12}$ Likewise, the prevalence detected at the CEC is higher than groups such as pregnant women $(0.26 \%)$, blood donors $(1.4 \%)$, and the general population (3.1\%). ${ }^{13-15}$

The highest seroprevalence of syphilis and active syphilis was among men and transgender people. It is possible that a high proportion of men attending the CEC are MSM, a group in which the HIV epidemic in Mexico is concentrated. ${ }^{15}$ In the case of men, the high frequency and incidence of active syphilis could be related to sexual practices, unprotected anal sex, a large number of sexual partners, and/or casual relationships. The transgender population had different vulnerabilities, namely being sex workers, unfavorable economic conditions, and/or stigma and discrimination. ${ }^{16}$

The highest frequency of active syphilis, as well as the highest incidence of syphilis, was found in 20-39year-old individuals. These data are consistent with the worldwide trend, which shows that the largest number of new STI cases occur in the young population. ${ }^{3}$ Thus, it is necessary to focus syphilis prevention and diagnosis programs in these groups. The presence of other STIs is a risk factor for acquisition and transmission of T. pallidum, as shown with HIV and HBV but not HCV (whose main route of transmission is parenteral rather than sexual). People living with HIV have a suppressed immune system, and this suppression increases their susceptibility to other STIs such as syphilis. ${ }^{17}$ People with HIV-syphilis coinfection have a higher risk of neurological complications, which may be more frequent, progress faster, and present atypical signs. These individuals may also have treatment failure or present serofast (individuals without syphilis but higher antibodies titers). ${ }^{18}$ On the other hand, syphilitic lesions increase the risk of acquiring and transmitting HIV; persons with syphilis have a 4-fold greater risk of acquiring HIV. ${ }^{19}$ This value is similar to current study among people attending the CEC.

The higher prevalence and incidence of syphilis among PLWH may be by the individual risk perception and their social networks. ${ }^{20}$ From the onset of antiretroviral treatment, the number of deaths decreased and life expectancy improved. Consequently, some PLWH continued with risky sexual behaviors, such as sex without a condom, anal sex, occasional sexual partners, concurrent sexual partners, and/or alcohol and illegal drug use. ${ }^{21}$

A high percentage of people had persistent infection, that is, they maintained similar levels of antibodies during the study period. Failures in syphilis treatment have been documented from $3 \%$ among people without HIV to $17 \%$ among PLWH. ${ }^{22}$ Furthermore, among PLWH, the antibody titers take longer to decrease (serofast patients) up to $25 \%$ of population. ${ }^{23}$ The CEC prescribes the treatment for syphilis; 
however, it is unknown if people receive the treatment, as most of the participants are sent to their family medical clinic for follow-up. Only the PLWH without social security are followed in the CEC.

There are few studies on the incidence of syphilis in Mexico. Among FSW, it was 4.31 cases, and among their stable sexual partners, it was 3.64 cases per 100 py. ${ }^{24}$ In current study, the VCT group had a similar incidence, 3.4 cases. However, the highest incidence was among the PLWH, with 16 cases per 100 py. The syphilis incidence was higher than detected in other countries, including Italy (1.94), Singapore (6.21), and Australia (5.47 per 100 py), all among PLWH. ${ }^{23,25,26}$ It was similar to the very high incidence of syphilis reported in Argentina (14.9 cases per 100 py). ${ }^{27}$ In addition to the high incidence, there was a high percentage of syphilis re-infection. In other countries, this re-infection has been reported and appears to be a risk factor for re-infection, being asymptomatic, living with HIV, being a MSM, and failure in case follow-up. ${ }^{28}$

In relation to limitations of current study, did not include sociodemographic, sexual behavior, and clinical information about participants; it was to difficult to know if all patients received the adequate treatment and follow up because there is no epidemiological surveillance information about others clinics; the prevalence of active infection without clinical information may be overestimating the prevalence especially among PLWH that presents serofast; and there was a potential selection bias in the dynamic cohort, due to the small number of cases with follow up.

\section{Conclusion}

Different vulnerable groups showed high prevalence of active syphilis, high incidence, and re-infections. The results confirm that it is necessary to allocate more resources for the detection, treatment and monitoring of syphilis cases, as established by WHO, which prioritizes three STIs: human papillomavirus, Neisseria gonorrhoeae, and T. pallidum, the latter with objectives of eliminating congenital syphilis and controlling syphilis in specific populations. ${ }^{29}$ The strategy should be focused specifically among vulnerable groups, such as FSW, IDUs, MSM, and transgender women. ${ }^{30}$

\section{Abbreviations}

People seeking HIV-1 voluntary counseling and testing: VCT

People in prison: PPr

People living with HIV: PLWH

World Health Organization: WHO

Female sex worker: FSW

Men who have sex with men: MSM

Page $12 / 18$ 
Intravenous drug users: IDUs

Clinica Especializada Condesa: CEC

Sexually transmitted infections: STI

Hepatitis B Virus: HBV

Hepatitis C Virus: HCV

Chemiluminescent microparticle immunoassay: CMIA

Venereal disease research laboratory: VDRL

chi-square: $\chi^{2}$

person years: py

Incidence rate ratios: IRR

Confidence intervals: $\mathrm{Cl}$

\section{Declarations}

- Ethics approval and consent to participate. The participants signed an informed consent letter. The study was approved by the ethics committee of the National Institute of Public Health

- Consent for publication. Not applicable

- Availability of data and materials. The datasets used and/or analyzed during the current study are available from the corresponding author on reasonable request.

- Competing interests. The authors declare that they have no competing interests

- Funding. Not funding

- Authors' contributions. Construct the dataset and statistical analyses. VRG. Laboratory analyses about syphilis, VHB, VHC and HIV. GCS Revised the dataset about laboratory results. AGR coordinate the laboratory analyses and the basic information about the assistant to "Clinica Especializada Condesa". AHO. Write the manuscript and analyzed the information. MOP Revised the paper. MASA Write the manuscript, statistical analyses and analyzed the information.

- Acknowledgements. Not applicable

- Authors' information. Not applicable

\section{References}


1. Newman L, Rowley J, Vander Hoorn S, Wijesooriya NS, Unemo M, Low N, et al. Global estimates of the prevalence and incidence of four curable sexually transmitted infections in 2012 based on systematic review and global reporting. PLoS One 2015;10

2. World Health Organization. Report on global sexually transmitted infection surveillance, 2018.

3. Sexually transmitted disease surveillance, 2017. Atlanta, GA: US Department of Health and Human Services, CDC; 2018.

4. European Centre for Disease Prevention and Control. Syphilis. In: ECDC. Annual epidemiological report for 2016. Stockholm: ECDC; 2018.

5. Herrera-Ortiz A, Uribe-Salas FJ, Olamendi-Portugal ML, García-Cisneros S, Conde-Glez CJ, SánchezAlemán MA. Analysis of the trend of syphilis acquired in Mexico during the period 2003-2013. Salud Publica Mex 2015;57:335-42.

6. Lynn WA, Lightman S. Syphilis and HIV: a dangerous combination. The Lancet Infect Dis. 2004;4:456-66.

7. Conde-González CJ, Valdespino JL, Juárez-Figueroa LA, Palma O, Olamendi-Portugal M, OlaizFernández G, et al. Prevalence of anti-treponemic antibodies and sociodemographic characteristics in adult Mexican population in 2000. Salud Publica Mex 2007;49 supl 3:S412-S420

8. Zoni AC, González MA, Sjögren HW. Syphilis in the most at-risk populations in Latin America and the Caribbean: a systematic review. Int J Infect Dis 201317.

9. Workowski KA, Bolan GA. Sexually Transmitted Diseases Treatment Guidelines, 2015. MMWR Recomm Rep 2015;64:34-48

10. Patterson TL, Semple SJ, Staines H, Lozada R, Orozovich P, Bucardo J, et al. Prevalence and correlates of HIV infection among female sex workers in 2 Mexico-US border cities. J Infect Dis 2008; 197:728-32.

11. Bautista-Arredondo S, González A, Servan-Mori E, Beynon F, Juarez-Figueroa L, Conde-Glez CJ, et al. A Cross-Sectional Study of Prisoners in Mexico City Comparing Prevalence of Transmissible Infections and Chronic Diseases with That in the General Population. PLoS One. 2015;10

12. National report on monitoring expanded commitments and targets to end AIDS (GAM Report). Mexico 2018

13. Yáñez-Alvarez I, Conde-González CJ, Uribe-Salas FJ, Olamendi-Portugal ML, García-Cisneros S, Sánchez-Alemán MA. Maternal/child seroprevalence of antibodies against Treponema pallidum at four general hospitals in the state of Morelos, Mexico. Arch Med Res 2012;43:571-7.

14. López-Balderas N, Hernández-Romano J, Cámara-Contreras M, Bravo-Sarmiento E, HernándezRomano PA. Trends in prevalence of HIV and syphilis in a central blood bank of Veracruz, Mexico. Transfus Apher Sci. 2019;58:94-99.

15. Bautista-Arredondo S, Colchero MA, Romero M, Conde-Glez CJ, Sosa-Rubí SG. Is the HIV epidemic stable among MSM in Mexico? HIV prevalence and risk behavior result from a nationally representative survey among men who have sex with men. PloS One 2013;8(9) 
16. Salas-Espinoza KJ, Menchaca-Diaz R, Patterson TL, Urada LA, Smith D, Strathdee SA, et al. HIV Prevalence and Risk Behaviors in Male to Female (MTF) Transgender Persons in Tijuana, Mexico. AIDS Behav 2017;21: 3271-3278.

17. Callegari FM, Pinto-Neto LF, Medeiros CJ, Scopel CB, Page K, Miranda AE. Syphilis and HIV coinfection in patients who attend an AIDS outpatient clinic in Vitoria, Brazil. AIDS and behavior. 2014;18 Suppl 1:104-9.

18. Johns DR, Tierney M, Felsenstein D. Alteration in the natural history of neurosyphilis by concurrent infection with the human immunodeficiency virus. N Engl J Med 1987;316:1569-72.

19. Galvin SR, Cohen MS. The role of sexually transmitted diseases in HIV transmission. Nat Rev Microbiol. 2004; 2:33-42.

20. Blair C, Passaro RC, Segura ER, Lake JE, Perez-Brumer AG, Sanchez J, et al. Sexual network characteristics of men who have sex with men with syphilis and/or gonorrhoea/chlamydia in Lima, Peru: network patterns as roadmaps for STI prevention interventions. Sex Transm Infect. 2019;95(5):336-341.

21. Cohen SE, Chew Ng RA, Katz KA, Bernstein KT, Samuel MC, Kerndt PR, et al. Repeat syphilis among men who have sex with men in California, 2002-2006: implications for syphilis elimination efforts. Am J Public Health 2012;102:e1-8

22. Ghanem KG, Erbelding EJ, Wiener ZS, Rompalo AM. Serological response to syphilis treatment in HIV-positive and HIV-negative patients attending sexually transmitted diseases clinics. Sex Transm Infect 2007; 83:97-101

23. Spagnuolo V, Poli A, Galli L, Nozza S, Bossolasco S, Cernuschi M, et al. Incidence and Predictors of Serological Treatment Response in Early and Late Syphilis Among People Living With HIV. Open Forum Infect Dis. 2019; 6(1)

24. Bazzi AR, Rangel G, Martinez G, Ulibarri MD, Syvertsen JL, Bazzi SA, et al. Incidence and Predictors of HIV and Sexually Transmitted Infections Among Female Sex Workers and Their Intimate Male Partners in Northern Mexico: A Longitudinal, Multilevel Study. Am J Epidemiol 2015; 1;181:723-31.

25. Ang LW, Wong CS, Ng OT, Leo YS. Incidence of syphilis among HIV-infected men in Singapore, 20062017: temporal trends and associated risk factors. Sex Transm Infect Published Online First: 01 August 2019.

26. Goddard SL, Poynten IM, Petoumenous K, Jin F, Hillman RJ, Law C, et al Prevalence, incidence and predictors of anal Chlamydia trachomatis, anal Neisseria gonorrhoeae and syphilis among older gay and bisexual men in the longitudinal Study for the Prevention of Anal Cancer (SPANC). Sex Transm Infect. 2019;95:477-483.

27. Bissio E, Cisneros V, Lopardo GD, Cassetti LI. Very high incidence of syphilis in HIV-infected men who have sex with men in Buenos Aires city: a retrospective cohort study. Sex Transm Infect 2017;93:323326.

28. Kenyon C, Lynen L, Florence E, Caluwaerts S, Vandenbruaene M, Apers L, et al. Syphilis reinfections pose problems for syphilis diagnosis in Antwerp, Belgium - 1992 to 2012. Euro Surveill. 
2014;19:20958.

29. World Health Organization. Global health sector strategy against sexually transmitted infections 2016-2021. Towards the end of STIs. WHO/RHR/16.09.

30. World Health Organization. Report on global sexually transmitted infection surveillance, 2018. Geneva: World Health Organization; 2018. Licence: CC BY-NC-SA 3.0 IGO.

\section{Figures}

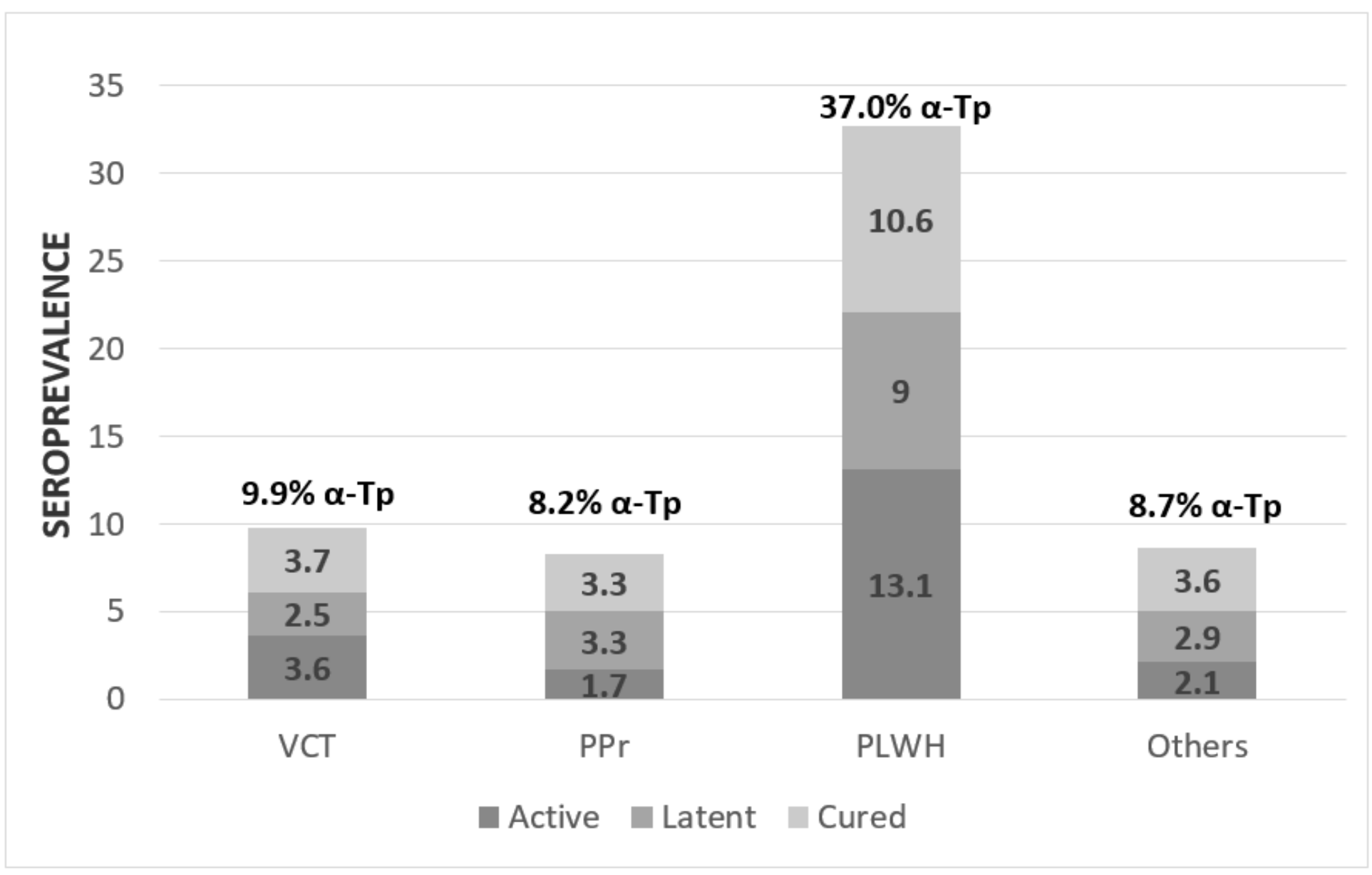

\section{Figure 1}

Seroprevalence of T. pallidum and active syphilis in populations attending a specialized HIV clinic in Mexico. a-Tp: antibodies against T. pallidum. Antibodies titers was not performed in $0.2 \%$ of people seeking HIV-1 voluntary counseling and testing (VCT), in $0.04 \%$ of people in prison (PPr), in $4.5 \%$ of people living with HIV (PLWH) and in $0.1 \%$ others. 


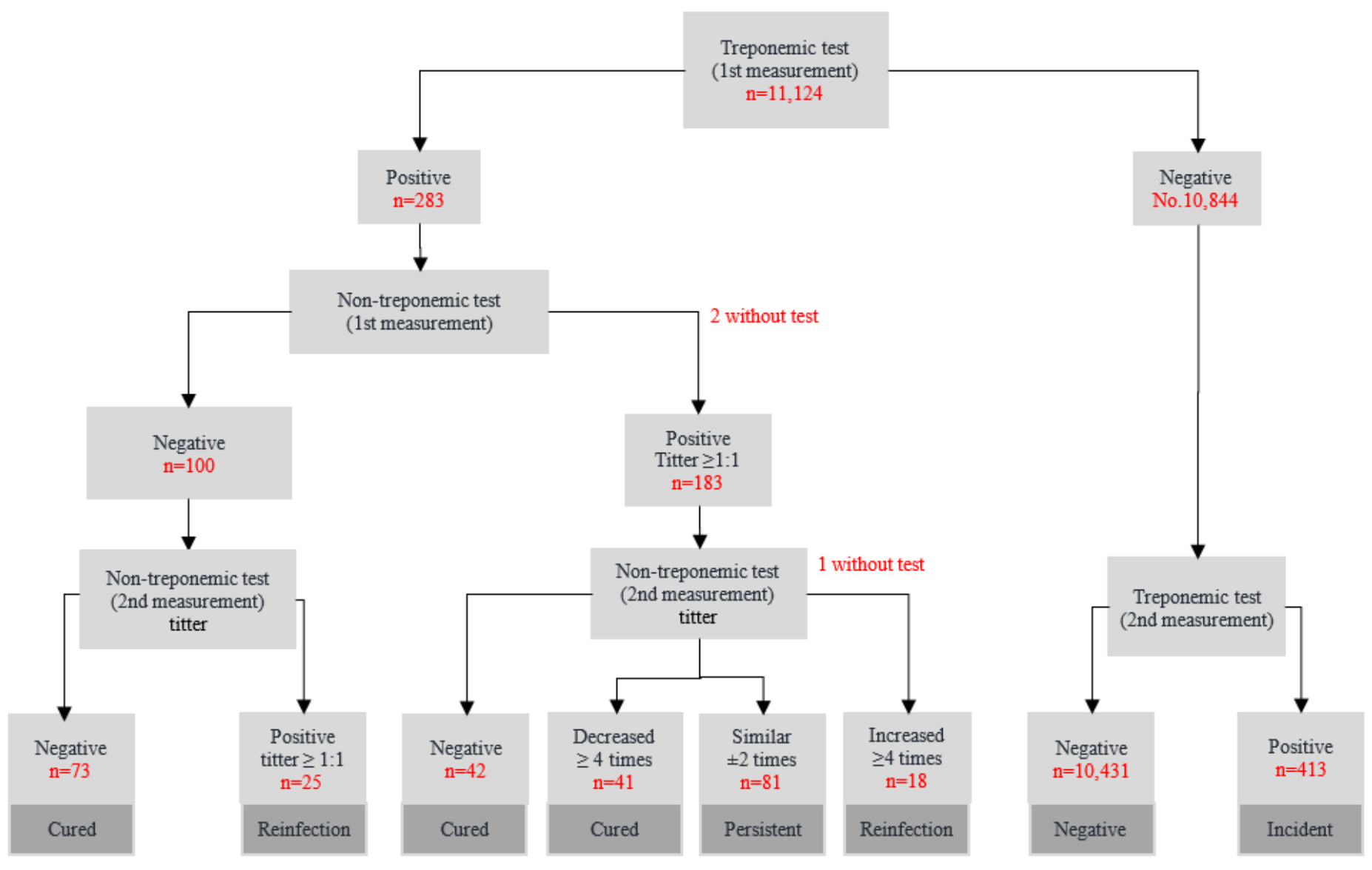

Figure 2

Retrospective dynamic cohort in populations attending a specialized HIV clinic in Mexico. 


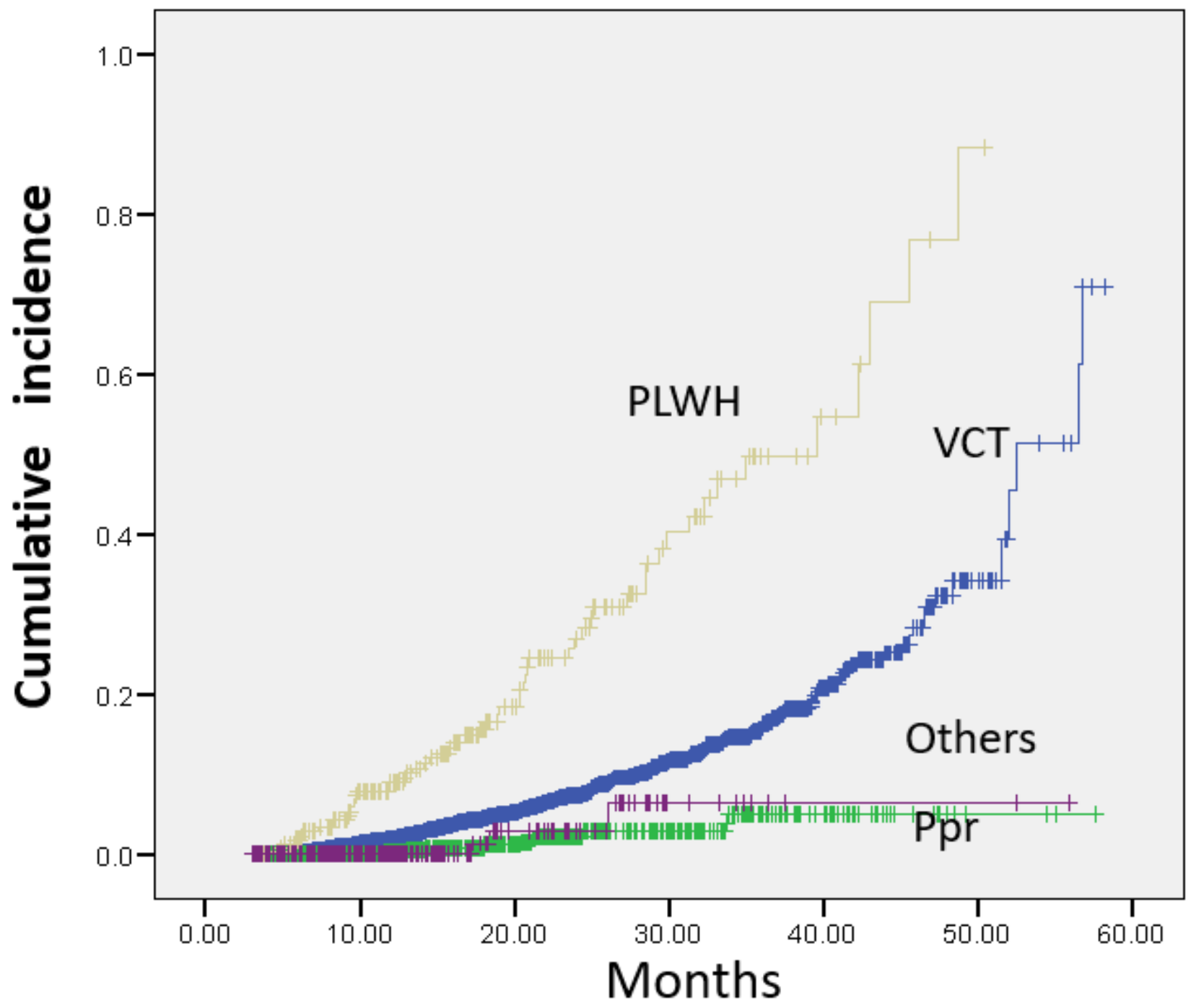

Figure 3

Cumulative incidence of T. pallidum in populations attending a specialized HIV clinic in Mexico. VCT: People seeking HIV-1 voluntary counseling and testing; PPr: people in prison; PLWH: people living with HIV; Others: patients from others clinics. 